\title{
Rehabilitation and Return to Sport After Arthroscopic Treatment of Femoroacetabular Impingement: A Review of the Recent Literature and Discussion of Advanced Rehabilitation Techniques for Athletes
}

\author{
Mark J. Holling, P.T., D.P.T., Scott T. Miller, P.T., M.S., S.C.S., C.S.C.S., and \\ Andrew G. Geeslin, M.D.
}

\begin{abstract}
The purpose of the article is to present an updated literature review, as well as describe our approach to rehabilitation and return to sports following hip arthroscopy for femoroacetabular impingement (FAI) with labral repair. A literature review was performed to identify articles published within the last 10 years that were focused on this topic. Relevant articles were reviewed, and reference lists were searched to identify additional articles. Findings were summarized for rehabilitation phases and return-to-sports assessment. Additionally, advanced rehabilitation topics are reviewed. Several systematic reviews and individual case series were identified. There is relative uniformity concerning the use of a four-phase approach for rehabilitation. However, there is inconsistency in terms of timing and criteria for ultimate return to sport. Advanced rehabilitation topics were reviewed, and description of their relevance at various rehabilitation phases was provided. A four-phase approach to rehabilitation following hip arthroscopy for FAI is widely used with general uniformity, although the timing and level of detail concerning assessment and readiness for return to sport are variable. Advanced rehabilitation techniques may be used in select patients returning to high-level activities.
\end{abstract}

\section{Introduction}

$\mathbf{T}$ he growth of arthroscopic treatment for management of femoroacetabular impingement (FAI) has been well documented. ${ }^{1}$ As with most sports medicine procedures, appropriate rehabilitation is believed to be a key component in a successful recovery. In spite of substantial expansion of surgical management, as well as literature concerning FAI, there is a relative paucity of articles on postoperative rehabilitation and return to

Armor Physical Therapy, Kalamazoo, Michigan (M.J.H., S.T.M.); and University of Vermont, Larner College of Medicine, Department of Orthopaedics and Rehabilitation, Burlington, Vermont (A.G.G.).

The authors report the following potential conflicts of interest or sources of funding: Dr. Geeslin reports: Arthroscopy: Editorial or governing board, Infographics Editor. Smith $\theta$ Nephew: Paid consultant; Paid presenter or speaker. Ossur: Paid presenter or speaker. Full ICMJE author disclosure forms are available for this article online, as supplementary material.

Received September 15, 2021; accepted November 9, 2021.

Address correspondence to Andrew G. Geeslin, M.D., 95 Carrigan Dr., Stafford Hall, 4th Floor, Department of Orthopaedics and Rehabilitation, Burlington,VT 05405,U.S.A.E-mail: andrew.geeslin@uvmhealth.org

(C) 2021 THE AUTHORS. Published by Elsevier Inc. on behalf of the Arthroscopy Association of North America. This is an open access article under the CC BY-NC-ND license (http://creativecommons.org/licenses/by-nc-nd/4.0/).

2666-061X/211325

https://doi.org/10.1016/j.asmr.2021.11.003 sport criteria. The purpose of the article is to present an updated literature review, as well as describe our approach to rehabilitation and return to sports following hip arthroscopy for FAI with labral repair.

\section{Methods}

A literature review was performed to identify articles published within the past 10 years that were focused on the topic of postoperative rehabilitation and return-to-sport assessments following hip arthroscopy for the treatment of FAI. Articles that focused on nonoperative treatment of FAI were not included in this review. Abstracts of selected articles were screened, and a full-text review of selected articles was performed. Additional articles were included on the basis of review of reference lists of selected articles, and our experience with rehabilitation of these patients.

Using the identified studies, rehabilitation and return to sport recommendations were analyzed and categorized. Return to sport recommendations were considered in the context of guidelines for other common surgical procedures, including anterior cruciate ligament (ACL) reconstruction. 


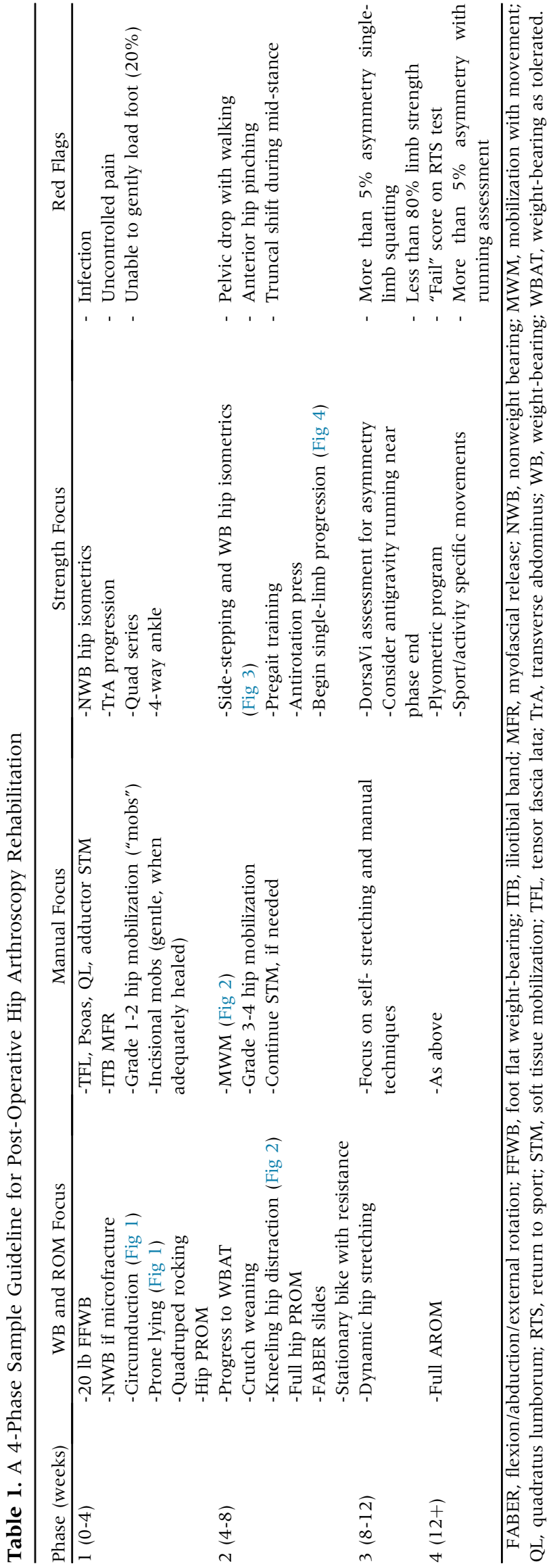

Special considerations for advanced rehabilitation were also reviewed in the context of the included articles, as well as our clinical practice. Advanced topics, including the use of patient-reported outcomes, video gait analysis, wearable motion sensor technology, and anti-gravity treadmill utilization are described along with the relevant literature and our clinical experience.

\section{Results}

Analysis and categorization of the recent hip arthroscopy rehabilitation literature revealed a mixture of 1) hip arthroscopy case series, including a focus on the rehabilitation guidelines; 2) return-to-sport testing and outcomes; and 3) systematic reviews of these topics.

\section{Rehabilitation Protocol}

Four relevant systematic reviews of hip arthroscopy rehabilitation were identified..$^{2-5}$ The systematic reviews identified that a structured program, including rehabilitation phases (typically four) are commonly used (Table 1). Additionally, range of motion and weightbearing restrictions vary between studies but are nearly universally included in rehabilitation guidelines.

Several relevant articles focusing on postoperative rehabilitation protocols were identified in the literature review. Amar et al. demonstrated improved outcomes with increased frequency and duration of physical therapy, as well as the importance of a home program when considering patients that would choose surgical treatment again. ${ }^{6}$ In a unique study, Cvetanovich et al. queried the International Society for Hip Arthroscopy (ISHA) surgeon database and reviewed their websites for rehabilitation protocols; even in this select group, considerable variability was identified. ${ }^{7}$

Together with two physical therapists, Domb described two-year outcomes and associated clinical guidelines for physical therapy following hip arthroscopy. ${ }^{8}$ Similar to other studies, a four-phase program was described, and a detailed chronological rehabilitation guideline was provided. The "HIPARTI" study described their rehabilitation approach, which focuses on targeting impairments expected following hip arthroscopy, namely strength, range of motion, and function. ${ }^{9}$ The authors recommended that rehabilitation should focus on providing care for patients with a chronic musculoskeletal disorder because features of early hip osteoarthritis are identified in many patients undergoing hip arthroscopy.

\section{Return to Sport}

As expected, much of the literature focuses on the ultimate outcome of return to desired athletic activities; for consistency, we will use the encompassing term "Return to Sport" (RTS). One systematic review ${ }^{10}$ and six individual studies ${ }^{11-16}$ focused on RTS results or 
Fig 1. Early right hip rehabilitation exercises for range of motion are shown. (A) Hip distraction: physical therapy (PT) provides gentle distraction of operative limb via a light traction force through the ankle. (B) Hip circumduction: PT places operative hip in $\sim 30-60^{\circ}$ of flexion to perform clockwise/ counterclockwise motion.

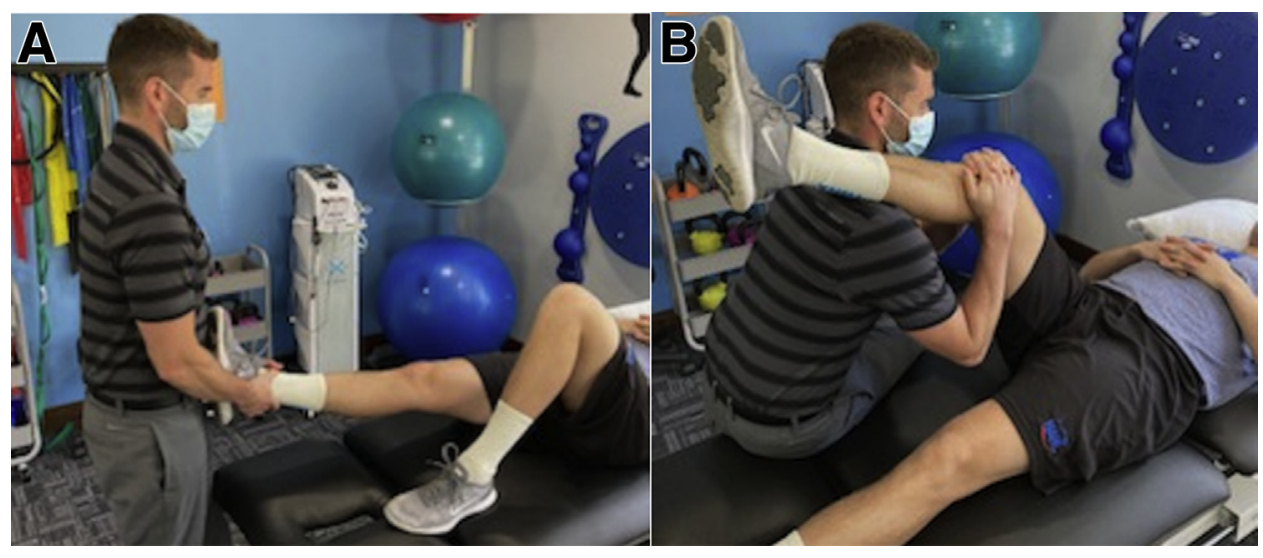

criteria. O'Connor reported the following items for sufficient RTS criteria: timeline, conditional criteria, description of measurements assessing the conditional criteria, and a specific rehabilitation protocol. ${ }^{10}$ Only two of the 22 studies in their systematic review satisfied the measures they deemed necessary for an effective RTS criteria.

Return to running was a primary focus of three included articles, and recommendations were made for this specific activity. ${ }^{11,13,14}$ Chen et al. allowed return to run after three months of physical therapy and the patient's demonstrated good stability while performing 30 consecutive single leg squats. ${ }^{11}$ Kraeutler et al. recommended similar timing, although a delay of an additional 6 weeks was recommended in the setting of cartilage restoration and/or acetabular dysplasia. ${ }^{13}$ They further recommended a focus on strength-maintaining exercises and a dynamic warm-up, as well as a threephase return-to-run program including 1) walking program, 2) quick response and plyometric routine
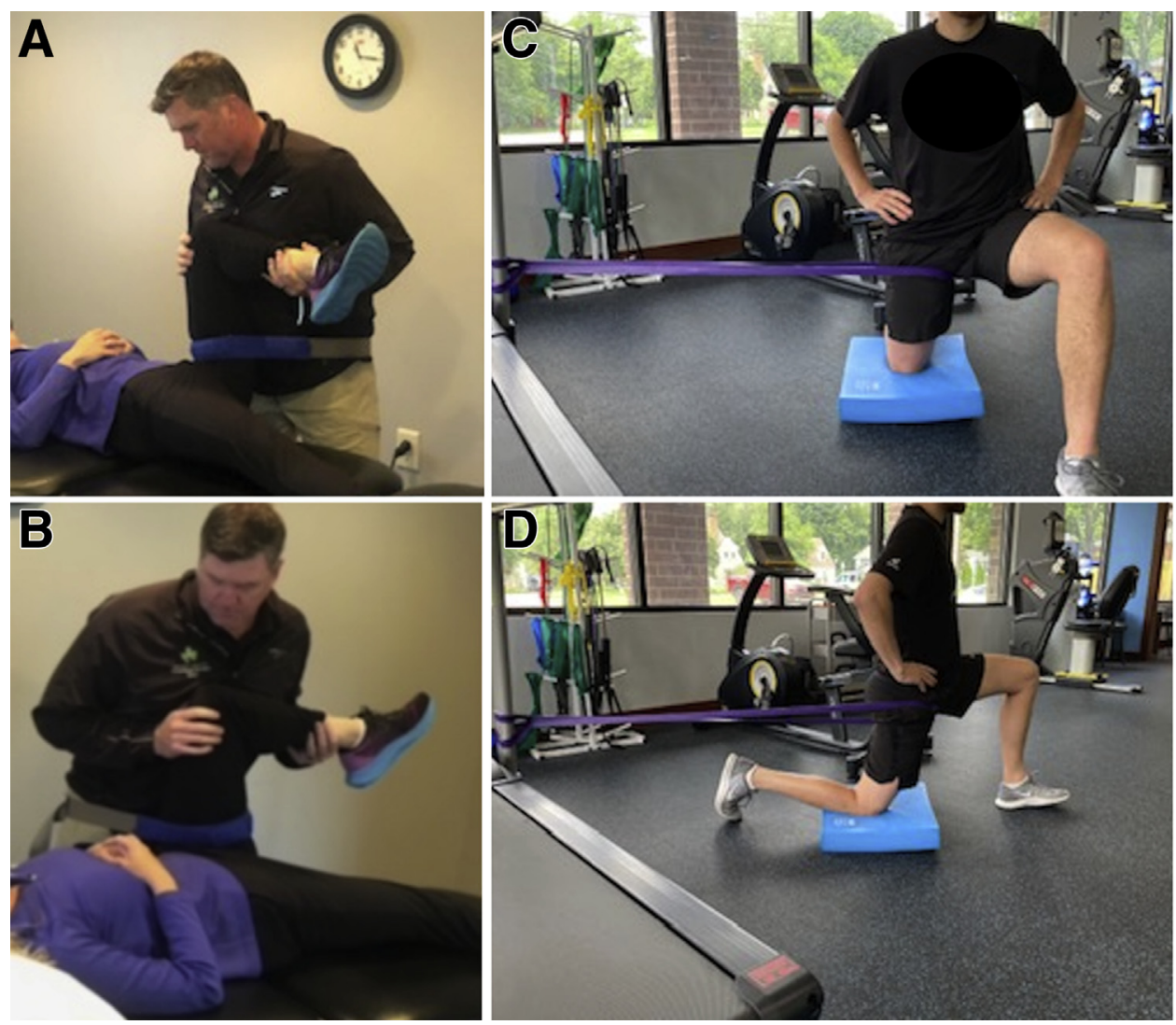

Fig 2. Gradual progression through a range of motion per the surgeon's guidelines is demonstrated with several techniques (A and B, operative left hip; C and D, operative right hip). Mobilization with movement (MWM) may be considered in consultation with the surgeon and may include gentle lateral distraction with hip flexion (A) and lateral distraction with hip internal rotation (B). (C) Band lateral distraction is performed with the patient kneeling through their operative hip with a band around the proximal thigh generating a laterally directed force. (D) Band posterior distraction is performed with the patient kneeling through their operative hip with a band around their proximal thigh generating a posteriorly directed force. 


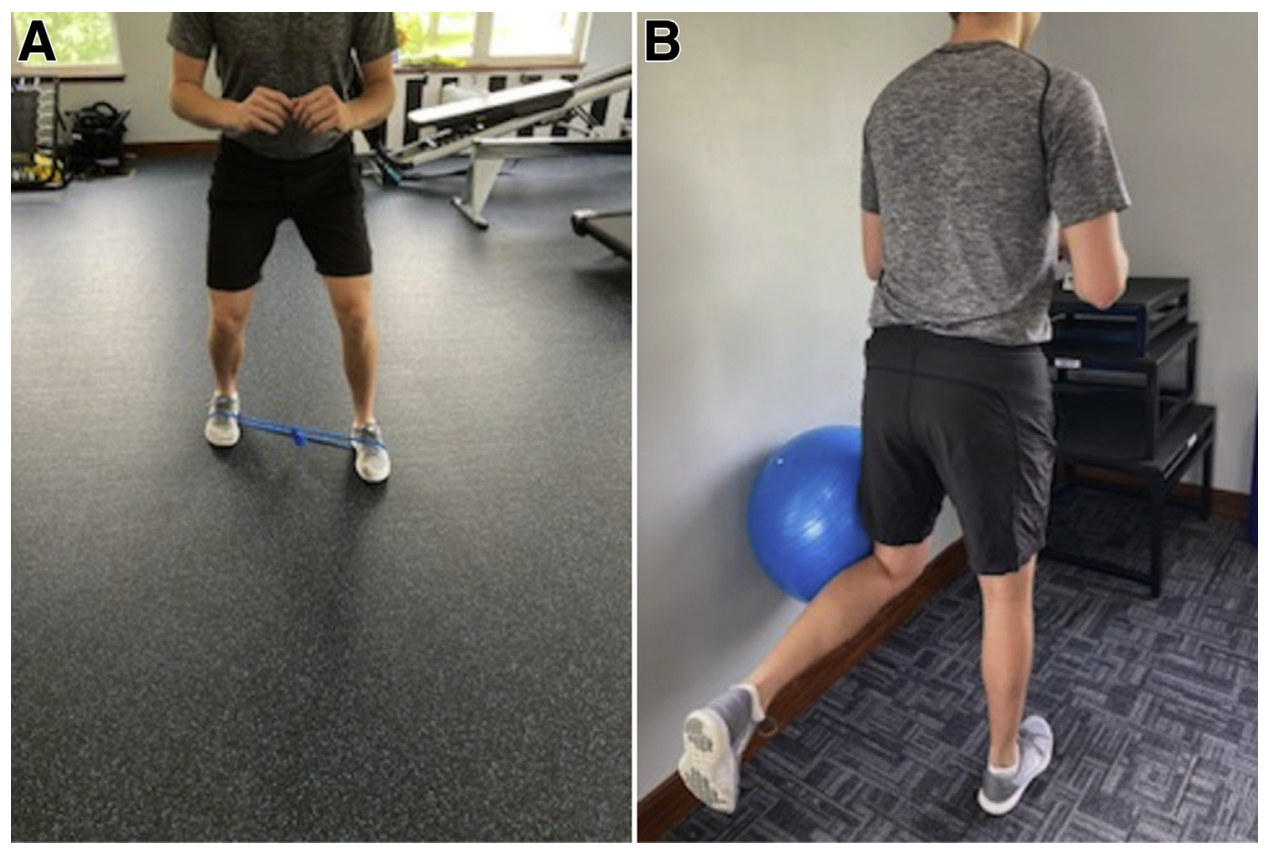

Fig 3. Weight-bearing exercises with an emphasis on the hip abductors are performed (operative right hip). (A) Resisted side steps as well as zig-zag (combined forward/diagonal movement) left and right are performed with an elastic band around the midfoot as tension is maintained. (B) A weight-bearing gluteus medius isometric is shown for the operative right hip as the patient's knee on the contralateral side supports the exercise ball against the wall, while maintaining an isometric contraction of the right hip gluteus medius.

with progression to a walk/jog program, and 3) return to distance running. Kuhns et al. also paid special attention to return to running during phase IV of their rehabilitation. ${ }^{14}$ Patients that successfully completed phases I-III were permitted to begin a return to running evaluation at 16 weeks on an anti-gravity treadmill and 20 weeks on a traditional treadmill.

\section{Discussion}

The most important findings of this review are the relative uniformity in the general rehabilitation guidelines with a four-phase approach, although there is substantial variability in criteria for return to sport. Therefore, a structured yet individualized plan with incorporated functional testing is recommended.

It is important for the surgeon to encourage an open line of communication with the physical therapy team. If surgery is elected, 1-2 preoperative sessions with the physical therapist will allow review of the surgeon's office visit notes, as well as a patient assessment for identification of preoperative functional deficits, such that a plan to address these deficits can be incorporated into the postoperative plan. The surgeon can support this communication by providing the therapist with a detailed operative report that includes a section focusing on the postoperative rehabilitation plan.

Specific factors that may influence rehabilitation timing and progression through an established postoperative rehabilitation guideline must be considered. Surgeon identified characteristics may include patient anatomy (e.g., borderline acetabular dysplasia) and tissue characteristics (e.g., complex labral tear, compromised labral integrity, large tear size, and capsular laxity). Certain combinations of these factors may support a delayed progression through a rehabilitation guideline, including delayed weight bearing and ROM progression.

Within the four-phase approach for rehabilitation, progression to the next phase is based on timing and requires mastery of activities in the previous phase. Phase 1 includes protection of the labral and capsular repair with limited weight-bearing and restricted range of motion. Phase 2 includes progression of weightbearing, return to walking, and early strengthening. Phase 3 includes advanced strengthening exercises and early agility drills with a consideration of return to running. Phase 4 builds upon the previous phases and individualizes agility drills, running goals, and functional testing for return to sport.

While there is general agreement of the overall structure of the phased rehabilitation approach, there is substantial variability with return to sport preparation and assessment following hip arthroscopy. Determination for return to sport following hip arthroscopy requires a multifactorial approach. Patient readiness, patient-reported outcomes (PRO), objective scoring, and specific sport testing are all important components to consider.

The ACL reconstruction literature serves as a reasonable guide when considering RTS, as readiness in this setting is felt to strongly influence reinjury rate. With regard to ACL reconstruction, there are numerous variations of RTS testing in the literature with some agreement on common key assessments (e.g., variations of single leg hop/jump). Rather than a defined testing protocol, recommendation for RTS following hip arthroscopy appears to rely on the absence of pain 
Fig 4. The single limb progression includes weight-bearing exercises through the patient's operative side (right hip) focusing on gluteus medius and core strength, as well as balance. (A) The single limb Pallof press is performed with the patient standing on their operative side and pressing forward against a firm resistant band anchored to their side. Sport cord taps are performed standing on the operative limb with an elastic cord placed around the waist, while tapping with the nonoperative foot and includes lateral tap (B), forward tap (C), and behind tap (D).
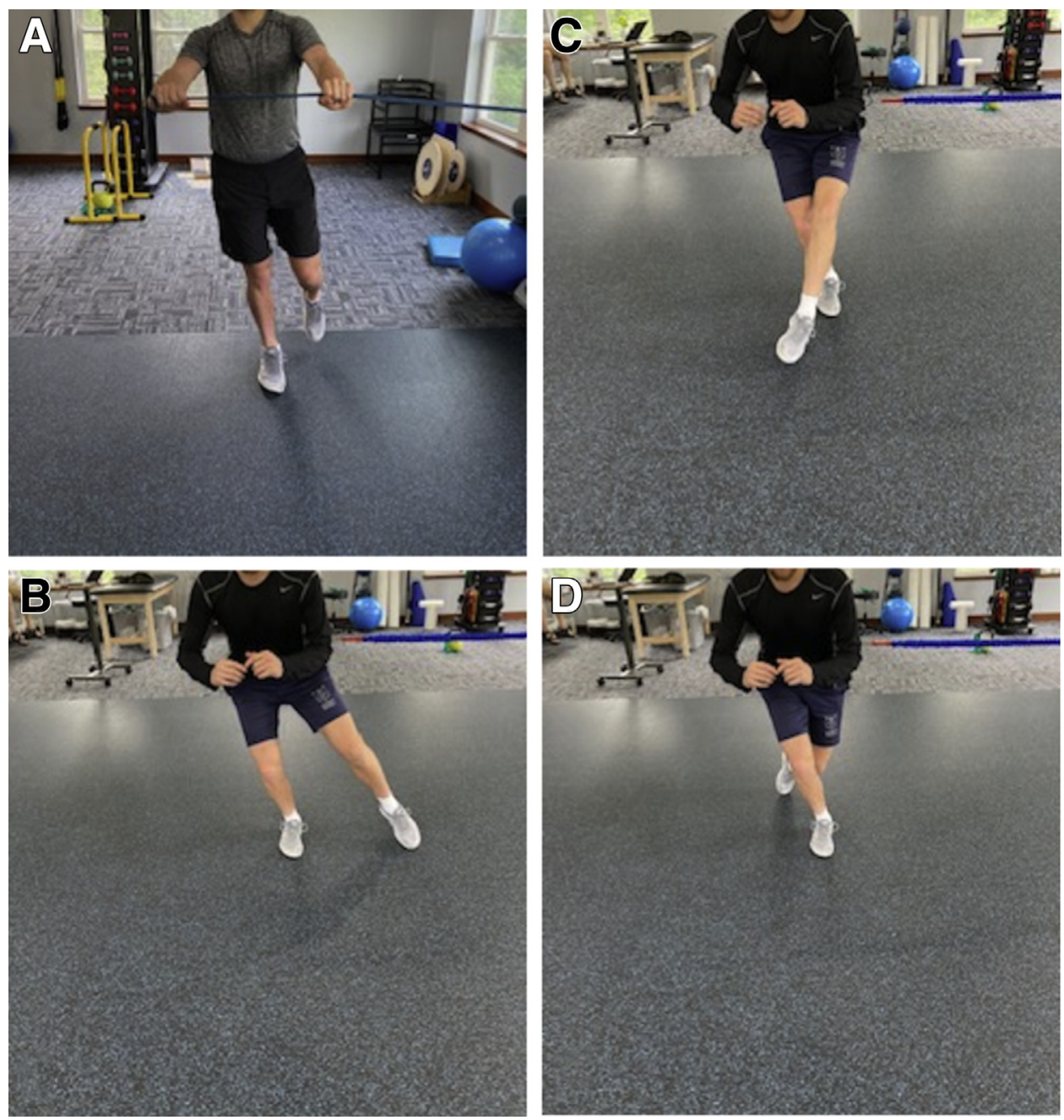

during a series of activities (including running, jumping, lateral agility drills, and single-leg squats) based on a survey of the hip. ${ }^{17}$

The reliance on general recommendations and absence of a universally accepted functional test issued for hip arthroscopy highlights the importance of fully understanding the patient's sport and occupational demands and tailoring the assessment to the individual patient. Recent literature recommends hip abduction/ adduction strength $>90 \%$ to uninvolved side for return to sport. $^{18}$ There are multiple RTS tests available, including the Hop Test, Y-balance, and Vail Hip Sports Test, although there is not yet consensus on an optimal test. One consideration from the ACL reconstruction literature that could be modified for hip arthroscopy is a comprehensive RTS test from University of Pittsburgh Medical Center (UPMC) Sports Medicine, which focuses on high-intensity impact and agility tests for return to sport (Table 2). ${ }^{19}$

The RTS test should be administered near the end of the 4th phase of rehabilitation, prior to clearing an athlete for unrestricted return to sports, especially if they are returning to a collision or contact sport. Although phase 4 typically begins 12 weeks postsurgery, it is important that the patient is meeting the proper criteria to be transitioned to phase 4 based on musculoskeletal objective measures rather than timing alone. The UPMC RTS test, like many other RTS tests, considers a passing score to be $90 \%$ of the uninvolved extremity, although it is our opinion that this number should instead target $>95 \%$. Upon completing the RTS test for any athlete, it is the responsibility of the physical therapist to provide the data to the orthopedic surgeon to make the final decision for clearance to return to unrestricted athletics.

\section{Special Considerations for Advanced Rehabilitation}

A reasonable target for all patients is return to activities of daily living, as well as a reasonable exercise routine to support a healthy lifestyle, and this can be assessed with patient-reported outcomes (PROs). Depending on the occupational and recreational goals, 
Table 2. Sample Return to Sports Testing Protocol
- -Single Broad Jump (Landing on One Foot)
- Triple Broad Jump (Landing on One Foot)
- Single Leg Forward Hop
- Timed 6-meter Single-Leg Hop
- Single-Leg Triple Hop
- Single-Leg Triple Crossover Hop
- Single-Leg Lateral Hop
- Single-Leg Medial Hop
- Single-Leg Medial Rotating Hop
- Single-Leg Lateral Rotating Hop
- Single-Leg Vertical Hop
- 10-Yard Lower Extremity Functional Test
- 10-Yard Proagility Run

Protocol from Joreitz et al., ${ }^{19}$ adapted from a protocol designed for anterior cruciate ligament reconstruction.

many patients may benefit from advanced rehabilitation techniques for return to high-level activities.

\section{Patient-Reported Outcomes}

Collection of PROs may be performed preoperatively and at regular intervals in follow-up. ${ }^{20}$ Some commonly used PROs include the modified Harris hip score (mHHS), hip outcomes score (HOS; activities of daily living and sports-specific subscales), nonarthritic hip score (NAHS), and lower extremity functional scale (LEFS); several other PROs are used and could also be considered. Tracking the scores and graphically displaying the results for the patient at selected intervals could be beneficial both in terms of patient education, as well as readiness for return to sport. However, a PRO more specific to the patient's sport or activity level may be more useful, and the Patient-Specific Functional Scale (PSFS) allows the physical therapist and patient to determine which activities or movements are most pertinent for return to their sport. The PSFS could also be issued at each session or weekly to better monitor a patient's progress.

\section{Video Gait Analysis}

Video gait analysis is a valuable assessment tool following hip arthroscopy and may reduce short- and long-term complications as patients return to running. Although 2-D video gait analysis has limitations compared to 3-D video gait analysis, it can provide excellent information for the running athlete, as it relates to key phases of gait and potential areas for improvement. Souza et al. describes 17 different variables that can be assessed for evidence-based videotaped running biomechanics analysis. ${ }^{21}$ Two key areas of emphasis to evaluate are landing pattern at initial contract and hip drop/pelvic obliquity. This can be accomplished with a more expensive multiview synchronized system or a tablet-based application. The authors of this study have used both types of systems and currently use an Apple iPad (Cupertino, CA) with the Technique by OnForm (OnForm, Shelton, CT).

\section{Running Mechanics}

Landing pattern, evaluated from a lateral view, describes foot position at initial contact, and broadly includes heel strike, midfoot strike, or forefoot strike. Tibial angle at initial contact can be simultaneously assessed. Magnitude of vertical ground reaction force (vGRF) may correlate with symptom development and perhaps reinjury; vGRF is greater when landing in front of the runner's center of mass (COM) compared to a landing closer to the COM. This influences the ability to generate proper muscle stiffness at impact throughout the entire lower extremity and may reduce injury at the ankles, knees, and hips.

Cadence (steps per unit time) manipulation is a method position of foot strike relative to COM. For example, a patient with a heel strike landing pattern with foot strike in front of their COM, increasing cadence may improve the runner's efficiency in transitioning to midstance and terminal stance, but also reduce their risk for injury. In a study in 2011 by Heiderscheit et al., a 5\% increase in cadence resulted in decreased vertical excursion of COM and braking impulse. $^{22}$ Furthermore, a $10 \%$ increase in step frequency resulted in decreased vGRF at the hip and knee, peak hip adduction angle, and peak hip adduction and internal rotation moments.

Hip drop and pelvic obliquity need to be assessed with single-leg standing and walking in routine rehabilitation. Although these may appear normal, higher-level activities, including running, may emphasize asymmetry due to muscle weakness and technique. With this insight, the clinician will appropriate dynamic activities to support hip functional stability during impact or single limb loading, which may decrease the risk of reinjury. Although normative data for acceptable pelvic drop in running is limited, Schache et al. reported an average pelvic drop of $5.4^{\circ} \pm 2.6^{\circ}$ just prior to takeoff. $^{23}$

\section{Wearable Motion Sensor Technology}

In recent years, sports rehabilitation centers have been employing motion sensor technology to monitor ROM, strength, force, power, and other valuable metrics to assist with clinical decision making. DorsaVi (London, UK) produces a wearable sensor applied to the patient's mid-leg to produce instant quantifying data based on the instructed movement and has been shown to be reliable and valid. This device can also measure a patient's degree of genu valgum/varus, tibial inclination, and speed during a single-leg squat test . The portable sensors also allow sport testing on the playing field for running analysis to measure ground reaction force, cadence, speed, initial peak acceleration, and ground contact time. The data are easily archived, enabling comparison to baseline and time-specific measurements. 


\section{Anti-Gravity Treadmill Considerations}

Incorporation of an antigravity treadmill (our practice currently uses a model from AlterG [AlterG, Fremont, CA]) into rehabilitation following hip arthroscopy and other lower extremity procedures has proven to be useful in our practice and can be considered throughout the rehabilitation process after initiation of weightbearing. We consider the following three segments: early mobility and gait retraining, preparation for return to running, and supplemental running). Individualized assessment of the patient's function by the physical therapist influences timing of progression.

\section{Early Mobility and Gait Retraining}

During the period of partial weight-bearing (PWB), an antigravity treadmill allows unloading to $20 \%$ of bodyweight and may be used to assist the patient in normalizing their gait pattern. Advanced communication with the surgeon is important to confirm their agreement with this approach during the PWB period. Early attention may aid in normalizing gait while avoiding an upper body trunk shift or a Trendelenburg gait pattern. It is believed that there is an elevated risk of symptoms related to the hip flexors during the first several postoperative weeks, and this must be evaluated. Garrison et al. discussed the importance of early motion exercises and reestablishing a symmetrical gait pattern to prevent concomitant stress throughout the lower extremity and spine, as well as a decreased risk for muscle imbalance and risk of lower crossed syndrome (characterized by gluteus inhibition due to a tight iliopsoas muscle-tendon unit). ${ }^{24}$ Compared to aquatherapy, an antigravity treadmill will simulate this unloaded environment with more specificity in gait, with the goal of allowing appropriate loading of the hip joint, while minimizing unnecessary stresses to healing tissue.

\section{Preparation for Return to Running}

Following gait normalization with walking, appropriate strengthening, and adequate soft tissue healing time, preparation for running may begin. According to our findings, 12 weeks is the most commonly referenced time for initiating return to running, providing that adequate rehabilitation progress and key functional milestones have been achieved. The authors of this study believe that it is important the patient demonstrates near normal strength prior to starting unloaded running in an antigravity treadmill and normal strength with proper gluteus recruitment prior to overground running. When starting this segment in an antigravity treadmill, a walk/run progression or a timebased running progression are frequently used. Progressively decreasing the percentage of body weight is a variable that can be used over a constant period of time as well. Patient-reported discomfort during and after running is an important consideration. Kraeutler et al. defined "acceptable" and "unacceptable" symptoms in response to running as a guideline for determining appropriate progression. ${ }^{13}$ Utilization of a gradual return to run progression may avoid setbacks secondary to poor technique and soft tissue inflammatory concerns. The goal of this segment is to have the patient nearly unweighted, before progressing to an overground program.

\section{Supplemental Running}

The goal of this phase is to assist the patient in getting back to their prior level of function as it relates to running. On the basis of our findings, a relatively high rate of return to running has been reported in some case series following hip arthroscopy, although intensity and distance are inconsistently reported. For example, Levy et al. reported a mean weekly distance of $9.5 \pm 6.5$ miles prior to injury that decreased to $6.4 \pm$ 5.8 miles postoperatively $(P<.01)$; despite decreased mileage, all 2-year outcomes scores improved significantly $(P<.001) .^{25}$ The use of an antigravity treadmill may allow patients to supplement their over-ground training program with some unloaded running with the goal to increase weekly mileage similar to a preoperative status, while minimizing the cumulative loading effect through the hip joint. Additionally, use of an antigravity treadmill provides an environment allowing patients to work on increasing speed in a potentially safer setting.

\section{Limitations}

The strength of recommendations of this review article is limited by the quality and type of evidence in the literature, and this field is primarily supported by level IV and V studies. A summary of recommendations for rehabilitation and progression of activity, including return to sport and running, is provided. Individualized detailed protocols for specific patient anatomy, surgical procedure details, and athletic activities are not yet able to be provided. Advanced rehabilitation techniques are reasonable in select patients in consultation with the surgeon, although detailed studies are not available to guide clinicians.

\section{Conclusions}

A four-phase approach to rehabilitation following hip arthroscopy for FAI is widely used with general uniformity, although the timing and level of detail concerning assessment and readiness for return to sport are variable. Advanced rehabilitation techniques may be used in select patients returning to high-level activities.

\section{References}

1. Cvetanovich GL, Chalmers PN, Levy DM, et al. Hip arthroscopy surgical volume trends and 30-day postoperative complications. Arthroscopy 2016;32:1286-1292. 
2. Ankem HK, Yelton MJ, Lall AC, et al. Structured physical therapy protocols following hip arthroscopy and their effect on patient-reported outcomes-a systematic review of the literature. J Hip Preserv Surg 2020;7:357-377.

3. Bistolfi A, Guidotti C, Aprato A, et al. Rehabilitation protocol following hip arthroscopy. A 2015 to 2020 systematic review. Am J Phys Med Rehabil 2021;100:958-965.

4. Grzybowski JS, Malloy P, Stegemann C, Bush-Joseph C, Harris JD, Nho SJ. Rehabilitation following hip arthroscopy. A systematic review. Front Surg 2015;2:21.

5. Reiman MP, Boyd J, Ingel N, Reichert A, Westhoven M, Peters S. There is limited and inconsistent reporting of postoperative rehabilitation for femoroacetabular impingement syndrome: A scoping review of 169 studies. J Orthop Sports Phys Ther 2020;50:252-258.

6. Amar E, Martin RL, Tudor A, Factor S, Atzmon R, Rath E. Midterm outcomes and satisfaction after hip arthroscopy are associated with postoperative rehabilitation factors. Orthop J Sports Med 2021;9:2325967120981888.

7. Cvetanovich GL, Lizzio V, Meta F, et al. Variability and comprehensiveness of North American online available physical therapy protocols following hip arthroscopy for femoroacetabular impingement and labral repair. Arthroscopy 2017;33:1998-2005.

8. Domb BG, Sgroi TA, VanDevender JC. Physical therapy protocol after hip arthroscopy: Clinical guidelines supported by 2-year outcomes. Sports Health 2016;8:347-354.

9. Heerey J, Risberg MA, Magnus J, et al. Impairment-based rehabilitation following hip arthroscopy: Postoperative protocol for the HIP ARThroscopy International Randomized Controlled Trial. J Orthop Sports Phys Ther 2018:48:336-342.

10. O'Connor M, Minkara AA, Westermann RW, Rosneck J, Lynch TS. Return to play after hip arthroscopy: A systematic review and meta-analysis. Am J Sports Med 2018;46:2780-2788.

11. Chen AW, Craig MJ, Yuen LC, Ortiz-Declet V, Maldonado DR, Domb BG. Five-year outcomes and return to sport of runners undergoing hip arthroscopy for labral tears with or without femoroacetabular impingement. Am J Sports Med 2019;47:1459-1466.

12. Hallberg S, Sansone M, Augustsson J. Full recovery of hip muscle strength is not achieved at return to sports in patients with femoroacetabular impingement surgery. Knee Surg Sports Traumatol Arthrosc 2020;28:1276-1282.

13. Kraeutler MJ, Anderson J, Chahla J, et al. Return to running after arthroscopic hip surgery: Literature review and proposal of a physical therapy protocol. J Hip Preserv Surg 2017;4:121-130.

14. Kuhns BD, Weber AE, Batko B, Nho SJ, Stegemann C. A four-phase physical therapy regimen for returning athletes to sport following hip arthroscopy for femoroacetabular impingement with routine capsular closure. Int J Sports Phys Ther 2017;12:683-696.

15. Lee S, Kuhn A, Draovitch P, Bedi A. Return to play following hip arthroscopy. Clin Sports Med 2016;35:637-654.

16. Tjong VK, Cogan CJ, Riederman BD, Terry MA. A qualitative assessment of return to sport after hip arthroscopy for femoroacetabular impingement. Orthop J Sports Med 2016;4:2325967116671940.

17. Domb BG, Stake CE, Finch NA, Cramer TL. Return to sport after hip arthroscopy: Aggregate recommendations from high-volume hip arthroscopy centers. Orthopedics 2014;37:e902-e905.

18. Thorborg K, Couppe C, Petersen J, Magnusson SP, Holmich P. Eccentric hip adduction and abduction strength in elite soccer players and matched controls: A cross-sectional study. Br J Sports Med 2011;45:10-13.

19. Joreitz R, Lynch A, Rabuck S, Lynch B, Davin S, Irrgang J. Patient-specific and surgery-specific factors that affect return to sport after ACL reconstruction. Int J Sports Phys Ther 2016;11:264-278.

20. Menge TJ, Briggs KK, Rahl MD, Philippon MJ. Hip arthroscopy for femoroacetabular impingement in adolescents: 10-year patient-reported outcomes. Am J Sports Med 2021;49:76-81.

21. Souza RB. An evidence-based videotaped running biomechanics analysis. Phys Med Rehabil Clin N Am 2016;27:217-236.

22. Heiderscheit BC, Chumanov ES, Michalski MP, Wille CM, Ryan MB. Effects of step rate manipulation on joint mechanics during running. Med Sci Sports Exerc 2011;43: 296-302.

23. Schache AG, Blanch P, Rath D, Wrigley T, Bennell K. Three-dimensional angular kinematics of the lumbar spine and pelvis during running. Hum Mov Sci 2002;21: 273-293.

24. Garrison JC, Osler MT, Singleton SB. Rehabilitation after arthroscopy of an acetabular labral tear. $N$ Am J Sports Phys Ther 2007;2:241-250.

25. Levy DM, Kuhns BD, Frank RM, et al. High rate of return to running for athletes after hip arthroscopy for the treatment of femoroacetabular impingement and capsular plication. Am J Sports Med 2017;45:127-134. 